\title{
Sharp Estimates of the Embedding \\ Constants for Besov Spaces $b_{p, q}^{s}, 0<p<1$
}

\author{
David E. Edmunds, W. Desmond Evans, \\ and Gyorgi E. KARADZHOV
}

\author{
School of Mathematics \\ Cardiff University \\ Senghennydd Road \\ Cardiff CF24 4AG, Wales - UK \\ davideedmunds@aol.com \\ EvansWD@cardiff.ac.uk
}

\author{
Institute of Mathematics and Informatics \\ Bulgarian Academy of Science \\ 1113 Sofia - Bulgaria \\ geremika@yahoo.com
}

Received: November 2, 2006

Accepted: March 22, 2007

\begin{abstract}
Sharp estimates are obtained for the rates of blow up of the norms of embeddings of Besov spaces $b_{p, q}^{s}$ in Lorentz spaces as the parameters approach critical values. In [8] the case $1 \leq p<\infty$ was investigated. The case $0<p<1$ of the present paper requires different methods as the pointwise estimates established are different and the interpolation argument used in [8] is no longer available.
\end{abstract}

Key words: Besov spaces, Lorentz spaces, embedding norms, rates of blow-up. 2000 Mathematics Subject Classification: 46E30, $46 \mathrm{E} 35$.

\section{Introduction}

In [8] we obtained sharp estimates for the rates of blow up of the norms of embeddings of Besov spaces in Lorentz spaces as the various parameters approached critical values. We describe this work briefly so as to provide some background and motivation for the present paper.

G. E. K. is grateful for a research grant from the School of Mathematics, Cardiff University. 
Let $p \in(0, \infty), q \in(0, \infty]$, denote by $\|\cdot\|_{p}$ the standard (quasi-)norm on $L_{p}=L_{p}\left(\mathbf{R}^{n}\right)$ and let $L_{p, q}$ be the usual Lorentz space, defined by the quasinorm

$$
\|f\|_{p, q}:= \begin{cases}\left(\int_{0}^{\infty}\left\{t^{1 / p} f^{*}(t)\right\}^{q} \frac{d t}{t}\right)^{1 / q}, & 0<q<\infty, \\ \sup _{0<t<\infty}\left\{t^{1 / p} f^{*}(t)\right\}, & q=\infty .\end{cases}
$$

Here $f^{*}$ is the non-increasing rearrangement of $f$, given by

$$
f^{*}(t)=\inf \left\{\lambda \geq 0: \mu_{f}(\lambda) \leq t\right\}, \quad t \geq 0,
$$

where $\mu_{f}$ is the distribution function of $f$, defined by

$$
\mu_{f}(\lambda)=\left|\left\{x \in \mathbf{R}^{n}:|f(x)| \geq \lambda\right\}\right|_{n},
$$

$|\cdot|_{n}$ denoting Lebesgue $n$-measure. We remark that all the spaces considered in this paper will consist of real- or complex-valued functions. Given any $s \in(0, \infty)$, the (homogeneous) Besov space $b_{p, q}^{s}$ is defined to be the set of all $f \in L_{p}\left(\mathbf{R}^{n}\right)$ with finite quasinorm

$$
\left\|f \mid b_{p, q}^{s}\right\|:=\left(\int_{0}^{\infty}\left\{t^{-s} \omega_{p}^{k}(t, f)\right\}^{q} \frac{d t}{t}\right)^{1 / q},
$$

(suitably interpreted when $q=\infty$ ), where $\omega_{p}^{k}(t, f):=\sup _{|h| \leq t}\left\|\Delta_{h}^{k} f\right\|_{p}$ is the $k^{\text {th }}$-order $L_{p}$-modulus of continuity of $f, \Delta_{h}^{k}$ being the $k^{\text {th }}$-order difference operator with step length $h$ defined recursively by

$$
\Delta_{h} f(x)=\Delta_{h}^{1} f(x):=f(x+h)-f(x), \quad \Delta_{h}^{k}:=\Delta_{h}^{1} \Delta_{h}^{k-1}, \quad(k \geq 2) .
$$

Here $k$ is any natural number greater than $s$; different choices of $k$ give equivalent quasinorms. We shall also write $b_{p, q}^{s}(k)$ rather than $b_{p, q}^{s}$ if it is desirable to stress the dependence on $k$. Moreover, we shall write $\omega_{p}(t, f)$ instead of $\omega_{p}^{1}(t, f)$.

It is well known (see [19]) that, if $p \in[1, \infty)$,

$$
b_{p, q}^{s} \hookrightarrow L_{r, q}, \quad \text { where } 1 / r=1 / p-s / n \text { and } 0<s<n / p,
$$

where by $X \hookrightarrow Y$ we mean that $X$ is continuously embedded in $Y$. In [8] sharp estimates were obtained of the rates of blow up of the embedding constant as $s \rightarrow(n / p)-$ in terms of

$$
b_{1}:=\sup _{f \neq 0}\|f\|_{r, q} /\left\|f\left|b_{p, q}^{s}\left\|, b_{2}:=\sup _{f \neq 0}\right\| f\left\|_{r} /\right\| f\right| b_{p, q}^{s}\right\| .
$$

Since $s \rightarrow n / p$ and $0<s<k$, we must have $n / p \leq k$. It was shown that as $s \rightarrow n / p<k$, with $1<p<\infty$ and $0<q \leq \infty$,

$$
b_{1} \approx r^{c}, \quad b_{2} \approx r^{(1-1 / q)_{+}}, \quad \text { where } \quad a_{+}=\max (a, 0) \text { and } c=\max (1,1 / q) .
$$


Here we use the notation $c \lesssim d$ or $d \gtrsim c$ to mean that $c$ is bounded above by a multiple of $d$, the multiple being independent of variables in $c$ and $d$; also, $c \approx d$ means that $c \lesssim d$ and $d \lesssim c$. If $k=n / p$ the results were different since $\left\|f \mid b_{p, q}^{s}\right\|$ may tend to infinity as $s \rightarrow n / p=k$. With $s=\sigma k, 0<\sigma<1$, we showed that if $1<p<\infty$, $k=n / p$, and $0<q \leq \infty$, then

$$
b_{p, q}^{k \sigma} \hookrightarrow(1-\sigma)^{-a+c} L_{r, q},
$$

where

$$
1 / r=(1-\sigma) / p, \quad a=\min (1 / p, 1 / q), \quad c=\max (1,1 / q) .
$$

In this, by $X \hookrightarrow \lambda Y$, where $X$ and $Y$ are quasi-Banach spaces and $\lambda>0$, we mean that there is a constant $C>0$, independent of $\lambda$, such that for all $f \in X, \lambda\|f|Y\|\leq C\| f|$ $X \|$. Special cases of these results were proved by Bourgain, Brezis, and Mironescu $([5,6])$, Maz'ya and Shaposhnikova $([15,16])$, Kolyada and Lerner $([11])$. For the embedding constants $b_{1}$ and $b_{2}$ of (2) we proved that if $1<p<\infty, k=n / p$, $q \in[1, \infty]$, and $1 / r=(1-\sigma) k / n$, then as $r \rightarrow \infty$,

$$
b_{1} \approx r^{1-1 / p}, b_{2} \approx r^{1-2 / p} .
$$

In this paper we extend the embedding (1) to cover the case in which $0<p<1$ and make corresponding extensions of (3) concerning the rates of blow up of the embedding constants. We show that

$$
b_{p, q}^{n / p-(1-\sigma) n} \hookrightarrow r^{-c} L_{r, q},
$$

where

$$
1 / r=1-\sigma, \quad 0<\sigma<1, \quad c=\max (1,1 / q), \quad 0<p<1,
$$

and that as $\sigma \rightarrow 1$, with $0<p<1, n / p<k$, and $0<q \leq \infty$,

$$
b_{1} \approx r^{c}, \quad b_{2} \approx r^{(1-1 / q)_{+}}, \quad \text { where } a_{+}=\max (a, 0) .
$$

The estimates (5) are also proved when $n / p=k$, but only in the case $n=1$; comparison with (4) shows that the cases $p<1$ and $p>1$ give entirely different results. The proofs make substantial use of the (nonlinear) spaces $L^{(\gamma)}(r, q)$ defined to be the set of all functions $f \in L_{\gamma}+L_{\infty}(0<\gamma \leq 1)$ such that $f^{*}(\infty)=0$ and

$$
\left\|f \mid L^{(\gamma)}(r, q)\right\|:= \begin{cases}\left\{\int_{0}^{\infty} t^{q / r}\left(\left(|f|^{\gamma}\right)^{* *}(t)-\left(|f|^{\gamma}\right)^{*}(t)\right)^{q / \gamma} \frac{d t}{t}\right\}^{1 / q}, & 0<q<\infty \\ \sup _{t>0} t^{1 / r}\left(\left(|f|^{\gamma}\right)^{* *}(t)-\left(|f|^{\gamma}\right)^{*}(t)\right)^{1 / \gamma}, & q=\infty\end{cases}
$$

is finite (see $[2,12,17])$. Here $g^{* *}(t)=t^{-1} \int_{0}^{t} g^{*}(s) d s$.

The paper concludes with a brief discussion of the supercritical case of embeddings of Besov spaces, by which we mean that the target space is $L_{\infty}$, and the proof of sharp embeddings of Besov spaces in the critical case $s=n / p$ (see also $[13,14]$ and for Sobolev spaces see $[1,9,12,17])$. 


\section{Preliminaries}

\subsection{The spaces $L^{(\gamma)}(r, q)$}

These spaces will play an important technical rôle in our arguments. For $0<q \leq$ $\infty$ and $-\infty<1 / r<1 / \gamma, 0<\gamma \leq 1$, the (nonlinear) space $L^{(\gamma)}(r, q)$ is defined (following $[2,12]$ and $[1,17]$ ) to be the family of all $f \in L_{\gamma}+L_{\infty}$ such that $f^{*}(\infty)=0$ and

$$
\left\|f \mid L^{(\gamma)}(r, q)\right\|:=\left\{\int_{0}^{\infty} t^{q / r}\left(\delta_{\gamma} f^{*}(t)\right)^{q} \frac{d t}{t}\right\}^{1 / q}<\infty
$$

(with the natural interpretation when $q=\infty$ ). Here

$$
\delta_{\gamma} f^{*}(t)=\left(\left(|f|^{\gamma}\right)^{* *}(t)-\left(|f|^{\gamma}\right)^{*}(t)\right)^{1 / \gamma}
$$

and $f^{* *}(t)=t^{-1} \int_{0}^{t} f^{*}(s) d s$. We recall that (see [3, Proposition 2.1.7]) $\left(|f|^{p}\right)^{*}=\left(f^{*}\right)^{p}$. Since $\frac{d}{d t}\left(|f|^{\gamma}\right)^{* *}(t)=t^{-1}\left\{\left(|f|^{\gamma}\right)^{*}(t)-\left(|f|^{\gamma}\right)^{* *}(t)\right\}$, it follows that

$$
\left(|f|^{\gamma}\right)^{* *}(t)=\int_{t}^{\infty}\left(\left(|f|^{\gamma}\right)^{* *}(s)-\left(|f|^{\gamma}\right)^{*}(s)\right) \frac{d s}{s} \quad \text { if } \quad f^{*}(\infty)=0 .
$$

Note that an application of l'Hôpital's rule to $f^{* *}(t)=t^{-1} \int_{0}^{t} f^{*}(s) d s$ shows that the condition $f^{*}(\infty)=0$ is equivalent to $f^{* *}(\infty)=0$. Moreover,

$$
L^{(\gamma)}(\infty, \gamma)=L_{\infty}
$$

The case $\gamma=1$ corresponds to the spaces $L(r, q)$ used in [8]. Routine inequalities show that

$$
L(r, q) \hookrightarrow L^{(\gamma)}(r, q) \quad \text { if } \quad-\infty<1 / r<\gamma<1, \quad \gamma>0,
$$

and

$$
L^{\left(\gamma_{1}\right)}(r, q) \hookrightarrow L^{(\gamma)}(r, q) \quad \text { if } \quad-\infty<1 / r<\gamma<\gamma_{1}, \quad \gamma>0,
$$

with an obvious extension of the use of the symbol $\hookrightarrow$.

Many properties of the spaces $L^{(\gamma)}(r, q)$ follow from those known for $L(r, q)$ together with the facts that $f \in L^{(\gamma)}(r, q)$ if and only if $|f|^{\gamma} \in L(r / \gamma, q / \gamma)$, and

$$
\left\|\left.f\left|L^{(\gamma)}(r, q)\|=\|\right| f\right|^{\gamma} \mid L(r / \gamma, q / \gamma)\right\|^{1 / \gamma} .
$$

In particular, we have the following embedding result immediately from [8, Lemma 1.1].

Lemma 1.1. Let $0<\gamma<r<\infty, \gamma \leq 1,0<q \leq \infty$ and put $c=\max (1 / \gamma, 1 / q)$. Then

$$
L^{(\gamma)}(r, q) \hookrightarrow r^{-c} L_{r, q}
$$


We note in passing that we can easily extend some of the results of [7] to the case when the functions involved are not necessarily integrable. First, we generalise the spaces $L^{(\gamma)}(r, q)$, replacing the weight $t^{q / r-1}$ by a general weight $w$. Thus we define the (nonlinear) space $L^{(\gamma)}(w, q)$ to be the set of all $f \in L_{\gamma}+L_{\infty}$ such that $f^{*}(\infty)=0$ and

$$
\left\|f \mid L^{(\gamma)}(w, q)\right\|:=\left\{\int_{0}^{\infty}\left(\delta_{\gamma} f^{*}(t)\right)^{q} w(t) d t\right\}^{1 / q}<\infty
$$

(with the usual understanding when $q=\infty$ ). The case $\gamma=1$ corresponds to the spaces considered in [7] and there denoted by $S_{q}(w)$. We remark that $f \in L^{(\gamma)}(w, q)$ if and only if $|f|^{\gamma} \in L(w, q / \gamma)$, and

$$
\left\|\left.f\left|L^{(\gamma)}(w, q)\|=\|\right| f\right|^{\gamma} \mid L(w, q / \gamma)\right\|^{1 / \gamma} .
$$

Now consider the corresponding generalised Lorentz spaces $\Lambda^{(\gamma)}(w, q)$ with quasi-norm

$$
\left\|f \mid \Lambda^{(\gamma)}(w, q)\right\|:=\left\{\int_{0}^{\infty}\left(\left(|f|^{\gamma}\right)^{* *}\right)^{q / \gamma} w(t) d t\right\}^{1 / q}
$$

(interpreted appropriately when $q=\infty$ ). As a consequence of the above remark and [7, Theorem 3.3] concerning the spaces $L(w, q)$ we see that

$$
L^{(\gamma)}(w, q)=\Lambda^{(\gamma)}(w, q) \text { for } q>\gamma \text { if and only if } w \in R B_{q / \gamma} .
$$

Here the class of weights $R B_{p}$ is defined in [7]: for $0<p<\infty, w \in R B_{p}$ if there is a constant $c>0$ such that, for all $r>0$,

$$
\int_{0}^{r} w(s) d s \leq c r^{p} \int_{r}^{\infty} w(s) s^{-p} d s
$$

Analogously, $L^{(\gamma)}(w, q)$ is a quasi-normed space for $q>\gamma$ if and only if $w \in R B_{q / \gamma}$.

\subsection{Pointwise estimates for the rearrangement}

Lemma 1.2. Let $k \in \mathbf{N}$ and suppose that $0<p<1$. Then for all $f \in L_{p}\left(\mathbf{R}^{n}\right)$,

$$
\left\{\left(|f|^{p}\right)^{* *}(t)-\left(|f|^{p}\right)^{* *}(2 t)\right\}^{1 / p} \lesssim t^{-1 / p} \omega_{p}\left(t^{1 / n}, f\right), \quad 0<t<\infty
$$

where $\omega_{p}=\omega_{p}^{1}$, and

$$
\begin{array}{r}
\left(|f|^{p}\right)^{* *}(t)-\left(|f|^{p}\right)^{* *}(2 t) \lesssim \int_{t}^{\infty} \int_{s_{k-1}}^{\infty} \ldots \int_{s_{2}}^{\infty} s_{1}^{-1}\left\{\omega_{p}^{k}\left(s_{1}^{1 / n}, f\right)\right\}^{p} \frac{d s_{1}}{s_{1}} \cdots \frac{d s_{k-1}}{s_{k-1}} \\
0<t<\infty, \quad k \geq 2 .
\end{array}
$$


Proof. First, note that

$$
\left(|f|^{p}\right)^{* *}(t)-\left(|f|^{p}\right)^{*}(t) \leq 2\left\{\left(|f|^{p}\right)^{* *}(t)-\left(|f|^{p}\right)^{* *}(2 t)\right\} .
$$

This follows from a more general inequality: see [20, Lemma 3.2]. Here we present another simple proof. From (6) it follows that

$$
\left(|f|^{p}\right)^{* *}(t)-\left(|f|^{p}\right)^{* *}(2 t)=\int_{t}^{2 t}\left\{\left(|f|^{p}\right)^{* *}(s)-\left(|f|^{p}\right)^{*}(s)\right\} \frac{d s}{s},
$$

and we now simply use the fact that $t \mapsto t\left\{\left(|f|^{p}\right)^{* *}(t)-\left(|f|^{p}\right)^{*}(t)\right\}$ is non-decreasing since its derivative is $-t \frac{d}{d t}\left(|f|^{p}\right)^{*}(t)$.

Let $t>0$ and let $B_{t}$ be the ball in $\mathbf{R}^{n}$ with centre 0 and volume $2 t$. Let $u \in B_{t}$. Since

$$
|f(x)| \leq\left|\Delta_{u} f(x)\right|+|f(x+u)|
$$

and hence

$$
|f(x)|^{p} \leq\left|\Delta_{u} f(x)\right|^{p}+|f(x+u)|^{p},
$$

we have, integrating with respect to $u$ over $B_{t}$,

$$
2 t|f(x)|^{p} \leq \int_{B_{t}}\left|\Delta_{u} f(x)\right|^{p} d u+\int_{0}^{2 t}\left(|f|^{p}\right)^{*}(s) d s .
$$

Now integrate with respect to $x$ over a subset $E$ of $\mathbf{R}^{n}$ with Lebesgue $n$-measure $t$ and take the supremum over all such sets $E$. This gives (see [3, p. 53, Proposition 2.3.3])

$$
\begin{aligned}
2 t\left\{\left(|f|^{p}\right)^{* *}(t)-\left(|f|^{p}\right)^{* *}(2 t)\right\} & \leq \int_{B_{t}}\left(\left|\Delta_{u} f\right|^{p}\right)^{* *}(t) d u \\
& =\frac{1}{t} \int_{B_{t}} \int_{0}^{t}\left(\left|\Delta_{u} f\right|^{p}\right)^{*}(s) d s d u \lesssim \frac{1}{t} \int_{B_{t}}\left\|\Delta_{u} f\right\|_{p}^{p} d u \\
& \leq \sup _{|u| \leq\left(2 t / \varpi_{n}\right)^{1 / n}}\left\|\Delta_{u} f\right\|_{p}^{p} \\
& =\left\{2 \omega_{p}^{1}\left(\left(2 t / \varpi_{n}\right)^{1 / n}, f\right)\right\}^{p}
\end{aligned}
$$

where $\varpi_{n}$ is the measure of the unit ball in $\mathbb{R}^{n}$. In view of $[4,(5.4 .5)$, p. 332], and the fact that $\omega_{p}^{1}$ is an increasing function, we see that (7) follows. Further, applying (6) to $\Delta_{u} f$ and using $(9)$ and (10), we obtain

$$
\begin{aligned}
\left(|f|^{p}\right)^{* *}(t)-\left(|f|^{p}\right)^{* *}( & \\
& \lesssim t^{-1} \int_{B_{t}} \int_{t}^{\infty}\left\{\left(\left|\Delta_{u} f\right|^{p}\right)^{* *}(s)-\left(\left|\Delta_{u} f\right|^{p}\right)^{* *}(2 s)\right\} \frac{d s}{s} d u
\end{aligned}
$$

Now (7) gives

$$
\left(|f|^{p}\right)^{* *}(t)-\left(|f|^{p}\right)^{* *}(2 t) \lesssim t^{-1} \int_{B_{t}} \int_{t}^{\infty} s_{1}^{-1}\left\{\omega_{p}\left(s_{1}^{1 / n}, \Delta_{u} f\right)\right\}^{p} \frac{d s_{1}}{s_{1}} d u .
$$


From [3, Lemma 5.4.11] and this we see that

$$
\left(|f|^{p}\right)^{* *}(t)-\left(|f|^{p}\right)^{* *}(2 t) \lesssim \int_{t}^{\infty} s_{1}^{-1}\left\{\omega_{p}^{2}\left(s_{1}^{1 / n}, f\right)\right\}^{p} \frac{d s_{1}}{s_{1}}
$$

which is the required estimate (8) for $k=2$. We proceed by induction. If (8) is valid for some $k \geq 2$, then from (11) we have

$$
\begin{aligned}
\left(|f|^{p}\right)^{* *}(t) & -\left(|f|^{p}\right)^{* *}(2 t) \\
& \lesssim t^{-1} \int_{B_{t}} \int_{t}^{\infty} \int_{s}^{\infty} \int_{s_{k-1}}^{\infty} \ldots \int_{s_{2}}^{\infty} s_{1}^{-1}\left\{\omega_{p}^{k}\left(s_{1}^{1 / n}, \Delta_{u} f\right)\right\}^{p} \frac{d s_{1}}{s_{1}} \cdots \frac{d s_{k-1}}{s_{k-1}} \frac{d s}{s} d u .
\end{aligned}
$$

The induction is established from [3, Lemma 5.4.11].

We also need the following variant of the estimate (8).

Lemma 1.3. Let $k \in \mathbf{N}, k \geq 2$ and suppose that $0<p<1$. Then for all $f \in L_{p}\left(\mathbf{R}^{n}\right)$,

$$
\begin{aligned}
\left(|f|^{p}\right)^{* *}(t)-\left(|f|^{p}\right)^{* *}(2 t) \lesssim \int_{t}^{1} \int_{s_{k-1}}^{1} \cdots \int_{s_{2}}^{1} s_{1}^{-1}\left\{\omega_{p}^{k}\left(s_{1}^{1 / n}, f\right)\right\}^{p} \frac{d s_{1}}{s_{1}} \cdots \frac{d s_{k-1}}{s_{k-1}} \\
+\left(1+|\log t|^{k-2}\right)\left\|f \mid L_{p}+L_{\infty}\right\|, \quad 0<t<1
\end{aligned}
$$

Proof. From (6) we have

$$
\left(|f|^{p}\right)^{* *}(t)=\int_{t}^{1}\left(\delta_{p} f^{*}\right)^{p}(u) d u / u+\left(|f|^{p}\right)^{* *}(1) .
$$

Since the $K$-functional for the pair $L_{p}, L_{\infty}$ satisfies

$$
K\left(t, f ; L_{p}, L_{\infty}\right) \approx\left(\int_{0}^{t^{p}}\left(f^{*}(s)\right)^{p} d s\right)^{1 / p}
$$

(see [4, Theorem 5.2.1]), we have

$$
\left\|f \mid L_{p}+L_{\infty}\right\|^{p}=K\left(1, f ; L_{p}, L_{\infty}\right)^{p} \approx \int_{0}^{1}\left(f^{*}(s)\right)^{p} d s=\left(|f|^{p}\right)^{* *}(1) .
$$

Thus

$$
\left(|f|^{p}\right)^{* *}(t) \approx \int_{t}^{1}\left(\delta_{p} f^{*}\right)^{p}(u) d u / u+\left\|f \mid L_{p}+L_{\infty}\right\|^{p} .
$$

Applying this to $\Delta_{u} f$ and using (9), (10), and (7) we obtain

$$
\left(\delta_{p} f^{*}\right)^{p}(t) \lesssim t^{-1} \int_{B_{t}} \int_{t}^{1} s_{1}^{-1}\left\{\omega_{p}\left(s_{1}^{1 / n}, \Delta_{u} f\right)\right\}^{p} \frac{d s_{1}}{s_{1}} d u+\left\|f \mid L_{p}+L_{\infty}\right\|^{p} .
$$


From [3, Lemma 5.4.11] and this we see that

$$
\left(\delta_{p} f^{*}\right)^{p}(t) \lesssim \int_{t}^{1} s_{1}^{-1}\left\{\omega_{p}^{2}\left(s_{1}^{1 / n}, f\right)\right\}^{p} \frac{d s_{1}}{s_{1}}+\left\|f \mid L_{p}+L_{\infty}\right\|^{p} .
$$

Repeating this procedure, we have from (13) and (14),

$$
\begin{aligned}
\left(\left|\Delta_{u} f\right|^{p}\right)^{* *}(t) \lesssim \int_{t}^{1}\left(\int_{s_{2}}^{1} s_{1}^{-1}\left\{\omega_{p}^{2}\left(s_{1}^{1 / n}, \Delta_{u} f\right)\right\}^{p} \frac{d s_{1}}{s_{1}}+\left\|f \mid L_{p}+L_{\infty}\right\|^{p}\right) \frac{d s_{2}}{s_{2}} \\
+\left\|f \mid L_{p}+L_{\infty}\right\|^{p} .
\end{aligned}
$$

Hence

$$
\left(\delta_{p} f^{*}\right)^{p}(t) \lesssim \int_{t}^{1} \int_{s_{2}}^{1} s_{1}^{-1}\left\{\omega_{p}^{3}\left(s_{1}^{1 / n}, f\right)\right\}^{p} \frac{d s_{1}}{s_{1}} \frac{d s_{2}}{s_{2}}+(1+|\log t|)\left\|f \mid L_{p}+L_{\infty}\right\|^{p} .
$$

In this way by induction we prove (12) for all $k \geq 2$.

\subsection{Embedding of Besov spaces in $L(r, q)$}

The main idea now is to reduce everything to the case $p=1$.

Theorem 1.4. Let $0<p<1,0<q \leq \infty$ and $0<s<k \in \mathbf{N}$. Then

$$
b_{p, q}^{s}(k) \hookrightarrow \beta^{c}(m-\beta)^{c} b_{1, q}^{\beta}(m),
$$

where $\beta=s-n / p+n, 0<\beta<m<k$, and $c=\max (1,1 / q)$.

Proof. First we prove that

$$
b_{p, 1}^{n / p-n}(k) \hookrightarrow L_{1}, \quad n / p-n<k .
$$

If $k=1$ this follows from (7), (9), and Lemma 1.1. If $k \geq 2$, then on substituting $s_{1} / s_{2}, s_{2} / s_{3}, \ldots, s_{k-2} / s_{k-1}, s_{k-1} / t$ for $s_{1}, s_{2}, \ldots, s_{k-2}, s_{k-1}$ in (8) we get

$$
\left(\delta_{p} f^{* *}(t)\right)^{p} \lesssim \int_{1}^{\infty} \cdots \int_{1}^{\infty} g^{p}\left(s_{1} s_{2} \cdots s_{k-1} t\right) \frac{d s_{1}}{s_{1}} \cdots \frac{d s_{k-1}}{s_{k-1}}, \quad g(u)=u^{-1 / p} \omega_{p}^{k}\left(u^{1 / n}, f\right) .
$$

Applying Minkowski's inequality, we find that

$$
\int_{0}^{\infty} \delta_{p} f^{* *}(t) d t \lesssim\left(\int_{1}^{\infty} \cdots \int_{1}^{\infty}\left(\int_{0}^{\infty} g\left(s_{1} s_{2} \cdots s_{k-1} t\right) d t\right)^{p} \frac{d s_{1}}{s_{1}} \cdots \frac{d s_{k-1}}{s_{k-1}}\right)^{1 / p},
$$

and after a change of variables we have

$$
\int_{0}^{\infty} \delta_{p} f^{* *}(t) d t \lesssim\left(\int_{1}^{\infty} \cdots \int_{1}^{\infty} s_{1}^{-p} \cdots s_{k-1}^{-p} \frac{d s_{1}}{s_{1}} \cdots \frac{d s_{k-1}}{s_{k-1}}\right)^{1 / p} \int_{0}^{\infty} t^{-1 / p} \omega_{p}^{k}\left(t^{1 / n}, f\right) d t
$$


which is exactly (16) in view of Lemma 1.1.

Application of the embedding (16) to the function $\Delta_{h}^{k} f$ for $|h| \leq t$ gives

$$
\left\|\Delta_{h}^{k} f\right\|_{1} \lesssim \int_{0}^{t} s^{n-n / p} \omega_{p}^{k}(s, f) d s / s+\int_{t}^{\infty} s^{n-n / p}\left\|\Delta_{h}^{k} f\right\|_{p} d s / s .
$$

Hence

$$
\omega_{1}^{k}(t, f) \lesssim \int_{0}^{t} s^{n-n / p} \omega_{p}^{k}(s, f) d s / s+t^{n-n / p} \omega_{p}^{k}(t, f)(n-n / p)^{-1} .
$$

Using also the estimate

$$
\int_{0}^{t} s^{n-n / p} \omega_{p}^{k}(s, f) d s / s \gtrsim t^{n-n / p} \omega_{p}^{k}(t, f),
$$

which follows easily from the monotonicity of $\omega_{p}^{k}(t, f)$ and the equivalence $\omega_{p}^{k}(t / 2, f) \approx$ $\omega_{p}^{k}(t, f)$, we obtain

$$
\omega_{1}^{k}(t, f) \lesssim \int_{0}^{t} s^{n-n / p} \omega_{p}^{k}(s, f) d s / s .
$$

This estimate shows that

$$
I:=\int_{0}^{\infty}\left\{t^{-\beta} \omega_{1}^{k}(t, f)\right\}^{q} d t / t \lesssim \int_{0}^{\infty} t^{-\beta q}\left(\int_{0}^{t} g(u) d u / u\right)^{q} d t / t,
$$

where $g(u)=u^{n-n / p} \omega_{p}^{k}(u, f)$.

If $q \geq 1$, then by a change of variables and Minkowski's inequality,

$$
\begin{aligned}
I & \lesssim\left(\int_{0}^{1}\left(\int_{0}^{\infty} t^{-\beta q} g(u t)^{q} d t / t\right)^{1 / q} d u / u\right)^{q} \\
& \lesssim\left(\int_{0}^{1} u^{\beta} d u / u\right)^{q} \int_{0}^{\infty} t^{-q(\beta+n / p-n)}\left(\omega_{p}^{k}(t, f)\right)^{q} d t / t .
\end{aligned}
$$

In other words,

$$
\left\|f\left|b_{1, q}^{\beta}(k)\left\|\lesssim \beta^{-c}\right\| f\right| b_{p, q}^{s}(k)\right\|, \quad s=\beta+n / p-n, \quad c=\max (1,1 / q) .
$$

If $0<q<1$, we put $h(t)=\int_{0}^{t} u^{n-n / p} \omega_{p}^{k}(u, f) d u / u$ and use integration by parts to obtain, assuming that the integrated terms play no part in the inequality,

$$
\begin{aligned}
I & \lesssim \int_{0}^{\infty} t^{-\beta q} h^{q}(t) d t / t \lesssim \beta^{-1} \int_{0}^{\infty} h^{q}(t) d t^{-\beta q} \\
& \lesssim \beta^{-1} \int_{0}^{\infty} t^{-\beta q} h^{q-1}(t) h^{\prime}(t) d t .
\end{aligned}
$$


Since

$$
h^{\prime}(t)=t^{n-n / p-1} \omega_{p}^{k}(t, f) \quad \text { and } \quad h(t) \gtrsim t^{n-n / p} \omega_{p}^{k}(t, f),
$$

we have (17) for this case also. It remains to justify the neglect of the integrated terms. As $\omega_{p}^{k}(u, f)$ is monotonic in $u$,

$$
\int_{t}^{2 t}\left(u^{-s} \omega_{p}^{k}(u, f)\right)^{q} d u / u \geq\left((2 t)^{-s} \omega_{p}^{k}(t, f)\right)^{q} \int_{t}^{2 t} d u / u
$$

and so

$$
\left(t^{-s} \omega_{p}^{k}(t, f)\right)^{q} \lesssim \int_{t}^{2 t}\left(u^{-s} \omega_{p}^{k}(u, f)\right)^{q} d u / u \rightarrow 0 \quad \text { as } t \rightarrow 0
$$

since $f \in b_{p, q}^{s}$. Hence by l'Hôpital's rule,

$$
\lim _{t \rightarrow 0} \frac{\int_{0}^{t} u^{n-n / p} \omega_{p}^{k}(u, f) d u / u}{t^{s-n / p+n}}=\lim _{t \rightarrow 0} \frac{t^{n-n / p-1} \omega_{p}^{k}(t, f)}{(s-n / p+n) t^{s-n / p+n-1}}=0 .
$$

Further, we show that (17) implies (15). We have to prove that

$$
b_{1, q}^{\beta}(k) \hookrightarrow(m-\beta)^{c} b_{1, q}^{\beta}(m), \quad k>m .
$$

To this end we use the Marchaud inequality (see [3, p. 332])

$$
\omega_{1}^{m}(t, f) \lesssim t^{m} \int_{t}^{\infty} \omega_{1}^{k}(u, f) u^{-1-m} d u, \quad m<k .
$$

Arguing as above (applying Minkowski's inequality if $q \geq 1$ and integrating by parts if $0<q<1$ ) we obtain (18).

Using also the estimate (see $[8,(13)]$ )

$$
f^{* *}(t)-f^{* *}(2 t) \lesssim t^{-1} \omega_{1}^{m}\left(t^{1 / n}, f\right), \quad m<n+2,
$$

we obtain the following Corollary.

Corollary 1.5. Let $0<p<1,0<q \leq \infty$, let $m, k \in \mathbf{N}$ and $s \in \mathbf{R}$ be such that $m<k, 0<s<k, n / p-n<s<n / p-n+m$, and let $1 / r=1 / p-s / n$, $\beta=s-n / p+n, \beta<m<n+2, c=\max (1,1 / q)$. Then

$$
b_{p, q}^{s}(k) \hookrightarrow \beta^{c}(m-\beta)^{c} L(r, q) .
$$




\section{Sharp embedding constants for Besov spaces}

\subsection{Subcritical case}

Here we establish the embedding (see [19])

$$
b_{p, q}^{s} \hookrightarrow L_{r, q}, \quad 1 / r=1 / p-s / n, \quad 0<s<n / p, \quad 0<p<1,
$$

and aim to find sharp rates of blow up for the embedding constants as $s \rightarrow n / p$. As explained in the introduction, this means that $n / p \leq k$. We remind the reader that the embedding constants are given by

$$
b_{1}:=\sup _{f \neq 0}\|f\|_{r, q} /\left\|f \mid b_{p, q}^{s}\right\| \quad \text { and } \quad b_{2}:=\sup _{f \neq 0}\|f\|_{r} /\left\|f \mid b_{p, q}^{s}\right\| .
$$

From Theorem 1.4 with $\beta=\sigma n$ and [10, Theorem 6] we obtain

Theorem 2.1. Let $0<p<1,0<q \leq \infty, n / p \leq k, 0<\sigma<1,1 / r=1-\sigma$ and put $c=\max (1,1 / q)$. Then as $\sigma \rightarrow 1$,

$$
b_{p, q}^{n / p-(1-\sigma) n} \hookrightarrow(1-\sigma)^{c} L_{r, q} .
$$

The estimates

$$
b_{1} \approx r^{c} \text { and } b_{2} \approx r^{(1-1 / q)+}
$$

hold if, in addition, either $n / p<k$ or $n=1$ and $p=1 / k$. The upper estimates for $b_{1}$ and $b_{2}$ implicit in (20) hold without these additional restrictions.

Proof. We only need to prove the relations (20). The embedding (19) gives the required estimate from above, and when $n / p<k$ the estimates from below can be proved in the same way as in [8]. The case $k=n / p$ is more difficult to settle, and at the moment we can deal only with the situation when $n=1$.

First suppose that $0<q \leq 1, n=1$ and $p=1 / k, k \in \mathbf{N}$. Define a function $f$ on $\mathbf{R}$ by

$$
f(x)= \begin{cases}x, & 0 \leq x \leq 1 / 2 \\ 1-x, & 1 / 2<x \leq 1 \\ 0, & \text { otherwise }\end{cases}
$$

We estimate $\Delta_{h}^{k} f(x)$ for small positive $h$ and observe that it is zero everywhere except in the intervals $(-k h, 0),(1 / 2-k h, 1 / 2)$, and $(1-k h, 1)$, in which we have the pointwise estimate

$$
\left|\Delta_{h}^{k} f(x)\right| \lesssim h .
$$

It follows that

$$
\int_{\mathbf{R}}\left|\Delta_{h}^{k} f(x)\right|^{p} d x \lesssim h^{1+p}
$$


so that

$$
\omega_{p}^{k}(t, f) \lesssim t^{k+1}
$$

Hence

$$
\left\|f \mid b_{p, q}^{1 / p-1+\sigma}\right\| \lesssim 1
$$

On the other hand,

$$
\|f \mid L(r, q)\| \approx r^{1 / q}
$$

and thus

$$
b_{1} \gtrsim r^{1 / q}
$$

Since $\|f\|_{r}=(r+1)^{-1 / r} / 2 \approx 1$, the desired lower estimate for $b_{2}$ also follows.

Next suppose that $1<q \leq \infty, n=1$ and $p=1 / k, k \in \mathbf{N}$. Again we obtain lower bounds for $b_{1}$ and $b_{2}$ by constructing a test function, but this time the argument is more delicate. Let $f: \mathbf{R} \rightarrow \mathbf{R}$ be even, zero outside the interval $(-1,1)$ and given by

$$
f(x)=j+2-2^{j+1} x, \quad x \in\left(2^{-j-1}, 2^{-j}\right], \quad j \in \mathbf{N}_{0}:=\mathbf{N} \cup\{0\} .
$$

We aim to estimate $\Delta_{h}^{2} f(x)$, and first take $h=-2^{-m}$ for a fixed large $m \in \mathbf{N}$. Since $f$ is even, we need only consider intervals in the positive half-line. For each $a \geq 0$ let $\delta_{a}$ be the interval $\left(2^{-a-1}, 2^{-a}\right)$. Let $x \in \delta_{j}$ for some $j \in \mathbf{N}_{0}$. We estimate $\int_{\delta_{j}}\left|\Delta_{h}^{2} f(x)\right|^{1 / 2} d x$ for $j>m-m_{0}$, where $m_{0}$ will be specified below. For any fixed $x \in \delta_{j}$ we can find $s_{0}, s_{1}$ such that $s_{0}>s_{1}>j$ and $|x+2 h| \in \delta_{s_{0}}$ while $|x+h| \in \delta_{s_{1}}$. In this way the interval $\delta_{j}$ may be covered by a union of corresponding intervals, where

and

$$
|f(x+2 h)-f(x+h)| \leq s_{0}-s_{1}+2 \quad \text { if } x \in \delta_{j} \cap \delta_{s_{0}} \cap \delta_{s_{1}}
$$

$$
|f(x+h)-f(x)| \leq s_{1}-j+2 \quad \text { if } x \in \delta_{j} \cap \delta_{s_{1}}
$$

Then we have

$$
\begin{aligned}
\int_{\delta_{j}}\left|\Delta_{h}^{2} f(x)\right|^{1 / 2} d x \leq & \sum_{s_{j}>j} \sum_{s_{0}>s_{1}}\left(s_{0}-s_{1}+2\right)^{1 / 2} 2^{-s_{0}-1} \\
& +\sum_{s_{j}>j}\left(s_{1}-j+2\right)^{1 / 2} 2^{-s_{1}-1} \\
\leq & \sum_{s_{j}>j} 2^{-s_{1}} \sum_{s_{0}>s_{1}}\left(s_{0}-s_{1}+2\right)^{1 / 2} 2^{-\left(s_{0}-s_{1}\right)-1} \\
& +\sum_{s_{j}>j}\left(s_{1}-j+2\right)^{1 / 2} 2^{-\left(s_{1}-j\right)-1} 2^{-j} \\
\lesssim & 2^{-j}
\end{aligned}
$$


Hence

$$
\sum_{j>m-m_{0}} \int_{\delta_{j}}\left|\Delta_{h}^{2} f(x)\right|^{1 / 2} d x \lesssim|h| .
$$

To handle the case $j \leq m-m_{0}$, let $x \in \delta_{j}$ and suppose that $x+2 h \in \delta_{j+1}$. Then $j \leq m-4$, and so we define $m_{0}=4$. For $j \leq m-4$, if $x \in\left(2^{-j-1}+2|h|, 2^{-j}\right)$, then all points $x, x+h, x+2 h$ lie in the same interval $\delta_{j}$, so that the second difference is zero. Thus we need to consider only the interval $\left(2^{-j-1}, 2^{-j-1}+2|h|\right)$. Then (if, say, $\left.x+h \in \delta_{j}\right)$

$$
\begin{aligned}
f(x+2 h)-2 f(x+h)+f(x)= & j+3-2^{j+2}(x+2 h) \\
& -2\left\{j+2-2^{j+1}(x+h)\right\}+j+2-2^{j+1} x \\
= & 1-2^{j+1} x-2^{j+2} h,
\end{aligned}
$$

and since $1<2^{j+1} x<1+2^{j+2}|h|$, we obtain

$$
\left|\Delta_{h}^{2} f(x)\right| \lesssim 2^{j-m}
$$

Hence

$$
\sum_{j \leq m-4} \int_{2^{-j-1}}^{2^{-j-1+2|h|}}\left|\Delta_{h}^{2} f(x)\right|^{1 / 2} d x \lesssim|h| .
$$

Now we suppose that $h=2^{-m}$ for a fixed large positive integer $m$. Let $2^{-j-1}<$ $x<2^{-j}, j \in \mathbf{N}_{0}$. First we estimate $\int_{2^{-j-1}}^{2^{-j}}\left|\Delta_{h}^{2} f(x)\right|^{1 / 2} d x$ for $j>m-2$. We can reduce the problem to that of estimating the first-order differences $f(x+2 h)-f(x+h)$ and $f(x+h)-f(x)$. Since $f$ is even, given any fixed $x \in\left(2^{-j-1}, 2^{-j}\right)$, there exist $s_{0}, s_{1}$, with $j>s_{0} \geq s_{1} \geq-1$, such that $2^{-s_{0}-1}<|x+h|<2^{-s_{0}}$ and $2^{-s_{1}-1}<|x+2 h|<$ $2^{-s_{1}}$; that is, $x+h \in \delta_{s_{0}}$ and $x+2 h \in \delta_{s_{1}}$. Then

$$
|f(x+2 h)-f(x+h)| \leq s_{0}-s_{1}+4 \quad \text { if } x \in \delta_{j} \cap \delta_{s_{0}} \cap \delta_{s_{1}}, s_{1} \geq-1
$$

and

$$
|f(x+h)-f(x)| \leq j-s_{0}+4 \quad \text { if } x \in \delta_{j} \cap \delta_{s_{0}} .
$$

We claim that $s_{0}, s_{1}>m-4$. To justify this, first let $j \geq s_{1}+2$. Then the inequalities $2^{-s_{1}-1}<x+2 h<2^{-s_{1}}$ and $2^{-j-1}<x+h<2^{-j}$ imply that $2^{-s_{1}-2} \leq h=2^{-m}$, so that $s_{1} \geq m-2$. If $j<s_{1}+2$, then $s_{1}+2>m-2$, and so $s_{1}>m-4$. The proof 
that $s_{0}>m-4$ is analogous. Hence

$$
\begin{aligned}
\sum_{j>m-2} \int_{\delta_{j}}\left|\Delta_{h}^{2} f(x)\right|^{1 / 2} d x \leq & \sum_{s_{1}>m-4} 2^{-s_{1}} \sum_{s_{0}>s_{1}}\left(s_{0}-s_{1}+4\right)^{1 / 2} 2^{-\left(s_{0}-s_{1}\right)} \\
& \times \sum_{j>s_{0}} 2^{-\left(j-s_{0}\right)} \\
& +\sum_{s_{0}>m-4} 2^{-s_{0}} \sum_{j>s_{0}}\left(j-s_{0}+4\right)^{1 / 2} 2^{-\left(j-s_{0}\right)} \\
\lesssim & 2^{-m}
\end{aligned}
$$

Now consider the case $j \leq m-2$. If $2^{-j-1}<x<2^{-j}-2 h$, then $\Delta_{h}^{2} f(x)=0$, and so we have only to consider the interval $2^{-j}-2 h<x<2^{-j}$, of length $2 h$. Let, for example, $x+h \in \delta_{j}$. Then

$$
\Delta_{h}^{2} f(x)=j+1-2^{j}(x+2 h)-2\left\{j+2-2^{j+1}(x+h)\right\}+j+2-2^{j+1} x,
$$

and so

$$
\left|\Delta_{h}^{2} f(x)\right| \leq\left|-1+2^{j} x+2^{j+1} h\right| \lesssim 2^{j} h=2^{j-m} .
$$

Hence

$$
\sum_{j \leq m-2} \int_{\delta_{j}}\left|\Delta_{h}^{2} f(x)\right|^{1 / 2} d x \lesssim h \sum_{j \leq m-2} 2^{-(m-j) / 2} \lesssim h .
$$

In this way we obtain the estimate

$$
\omega_{p}^{k}(t ; f) \lesssim t^{k} \quad \text { if } \quad 0<t<1, \quad k=1 / p,
$$

which gives $\left\|f \mid b_{p, q}^{k-1 / r}\right\| \lesssim r^{1 / q}$. It remains to check that $\|f\|_{r} \approx r$. In fact,

$$
\|f\|_{r}^{r}=\sum_{j \geq 0} \int_{\delta_{j}}\left(j+2-2^{j+1} x\right)^{r} d x=\sum_{j \geq 0} 2^{-j-1} \int_{1}^{2}(j+2-s)^{r} d s \lesssim \sum_{j \geq 0} 2^{-j} j^{r} \lesssim r ! .
$$

On the other hand, for $j \approx r$,

$$
\|f\|_{r}^{r} \geq \int_{\delta_{j}}\left(j+2-2^{j+1} x\right)^{r} d x=2^{-j-1} \int_{1}^{2}(j+2-s)^{r} d s \gtrsim 2^{-r} r^{r}
$$

so that $b_{1} \gtrsim r$. The estimates for $b_{2}$ follow as before.

\subsection{Supercritical case}

When $s>n / p$ we know (see [21]) that

$$
B_{p, q}^{s} \hookrightarrow L_{\infty},
$$


where $B_{p, q}^{s}$ is the inhomogeneous Besov space, defined by means of Fourier decompositions. The problem of finding sharp rates of blow up for the corresponding embedding constants as $s \rightarrow(n / p)+$ was considered recently by Triebel [22], who dealt not only with Besov but also with Lizorkin-Triebel spaces, both types of spaces being considered on bounded Lipschitz domains. Here we consider the same problem for the slightly larger Besov spaces $\tilde{b}_{p, q}^{s}, 0<p<1$ (the case $1 \leq p<\infty$ was considered in [8]), defined by means of the quasi-norm

$$
\left\|f\left|\tilde{b}_{p, q}^{s}\left\|:=\left(\int_{0}^{\infty}\left\{t^{-s} \omega_{p}^{k+1}(t, f)\right\}^{q} \frac{d t}{t}\right)^{1 / q}+\right\| f\right| L_{p}+L_{\infty}\right\|, \quad n / p<s<k .
$$

Using monotonicity, we can replace the above integral by a sum and conclude that the scale $\tilde{b}_{p, q}^{s}$ is increasing with respect to $q$. It turns out that the results concerning the embedding constants are the same as in [22]. Let

$$
b_{3}:=\sup _{f \neq 0}\|f\|_{\infty} /\left\|f \mid \tilde{b}_{p, q}^{s}\right\| .
$$

Theorem 2.2. Let $0<p<1,0<q \leq \infty$, and $\sigma>0$. Then as $\sigma \rightarrow 0$,

$$
\tilde{b}_{p, q}^{n / p+\sigma} \hookrightarrow \sigma^{(1-1 / q)}+L_{\infty}
$$

Proof. First we prove that in the definition of the space $\tilde{b}_{p, q}^{s}$ we can replace the weaker quasi-norm of $L_{p}+L_{\infty}$ by the stronger norm of $L_{1}+L_{\infty}$. To this end we establish the embedding

$$
\tilde{b}_{p, q}^{n / p} \hookrightarrow L_{1}+L_{\infty}
$$

In fact,

$$
\begin{aligned}
\left\|f \mid L_{1}+L_{\infty}\right\| & =\int_{0}^{1} f^{*}(s) d s \leq \int_{0}^{1}\left(t^{-1} \int_{0}^{t}\left(f^{*}(u)\right)^{p} d u\right)^{1 / p} d t \\
& =\int_{0}^{1}\left(\left(|f|^{p}\right)^{* *}\right)^{1 / p} d t \\
& \leq \int_{0}^{1}\left(\int_{t}^{1}\left(\delta_{p} f^{*}(s)\right)^{p} d s / s+\left\|f \mid L_{p}+L_{\infty}\right\|^{p}\right)^{1 / p} d t \\
& \lesssim \int_{0}^{1}\left(\int_{t}^{1}\left(\delta_{p} f^{*}(s)\right)^{p} d s / s\right)^{1 / p} d t+\left\|f \mid L_{p}+L_{\infty}\right\| \\
& \lesssim \int_{0}^{1}\left(\int_{1}^{1 / t}\left(\delta_{p} f^{*}(s t)\right)^{p} d s / s\right)^{1 / p} d t+\left\|f \mid L_{p}+L_{\infty}\right\| \\
& \lesssim\left(\int_{1}^{\infty}\left(\int_{1}^{1 / s}\left(\delta_{p} f^{*}(s t)\right)^{p} d t\right)^{p} d s / s\right)^{1 / p}+\left\|f \mid L_{p}+L_{\infty}\right\| \\
& \lesssim\left(\int_{1}^{\infty} s^{-p} d s / s\right)^{1 / p} \int_{0}^{1} \delta_{p} f^{*}(t) d t+\left\|f \mid L_{p}+L_{\infty}\right\| .
\end{aligned}
$$


Thus

$$
\left\|f\left|L_{1}+L_{\infty}\left\|\lesssim \int_{0}^{1} \delta_{p} f^{*}(t) d t+\right\| f\right| L_{p}+L_{\infty}\right\| .
$$

Now we use the estimates (9) and (12). Applying also Minkowski's inequality as above, we obtain the estimate

$$
\begin{aligned}
\left\|f \mid L_{1}+L_{\infty}\right\| \lesssim & \int_{0}^{1}\left(\int_{t}^{1} \int_{s_{k-1}}^{1} \ldots \int_{s_{2}}^{1} s_{1}^{-1}\left(\omega_{p}^{k}\left(s_{1}^{1 / n}, f\right)\right)^{p} \frac{d s_{1}}{s_{1}} \cdots \frac{d s_{k-1}}{s_{k-1}}\right)^{1 / p} d t \\
& +\left\|f \mid L_{p}+L_{\infty}\right\| \int_{0}^{1}\left(1+|\log t|^{(k-2) / p}\right) d t \\
& \lesssim\left(\int_{1}^{\infty} \cdots \int_{1}^{\infty} s_{1}^{-p} \cdots s_{k-1}^{-p} \frac{d s_{1}}{s_{1}} \cdots \frac{d s_{k-1}}{s_{k-1}}\right)^{1 / p} \int_{0}^{1} u^{-1 / p} \omega_{p}^{k}\left(u^{1 / n}, f\right) d u \\
& +\left\|f \mid L_{p}+L_{\infty}\right\| .
\end{aligned}
$$

Hence (21) is established for $q=\infty$, and so also for any $q$ by monotonicity. The same proof gives

$$
\tilde{b}_{p, q}^{n / p+\sigma} \hookrightarrow L_{1}+L_{\infty}
$$

uniformly for $\sigma \geq 0$. In other words, we have

$$
\tilde{b}_{p, q}^{n / p+\sigma}=b_{p, q}^{n / p+\sigma} \cap\left(L_{1}+L_{\infty}\right),
$$

uniformly for $\sigma \geq 0$.

Moreover, from Theorem 1.4 with $s=n / p+\sigma, \beta=\sigma+n, m=n+1$ and small $\sigma>0$, together with (22), we have

$$
\tilde{b}_{p, q}^{n / p+\sigma} \hookrightarrow \tilde{b}_{1, q}^{n+\sigma}(n+1), \quad 0<p<1,
$$

uniformly with respect to $\sigma$. Finally, [8, Theorem 4.1] gives

$$
\tilde{b}_{1, q}^{n+\sigma} \hookrightarrow \sigma^{(1-1 / q)+} L_{\infty}
$$

As a consequence we have

Corollary 2.3. Under the conditions of the last theorem,

$$
b_{3} \approx \sigma^{-(1-1 / q)+} .
$$

Part of this follows from [22], since $B_{p, q}^{s} \hookrightarrow \tilde{b}_{p, q}^{s}$, uniformly with respect to $s \rightarrow$ $(n / p)+($ see $[21$, p. 110]). 


\subsection{Critical case}

Here we derive sharp embeddings in the critical case $s=n / p, 0<p<\infty$. First, from Corollary 1.5, with $s=n / p, \beta=n$ and $m=n+1$ if $0<p<1$, and from $[8$, Lemma 2.2] if $p \geq 1$, it follows that

$$
b_{p, q}^{n / p} \hookrightarrow L(\infty, q), \quad 0<p<\infty, \quad 0<q \leq \infty .
$$

If $0<q \leq 1$, then $L(\infty, q) \hookrightarrow L_{\infty}$, and so (23) is a sharpening of the well-known embeddings [21]. When $q>1$ we can replace $L(\infty, q)$ by a linear and larger space. Let $L^{\infty, q}(\log L)_{-1}$ be the space with norm

$$
\left\|f \mid L^{\infty, q}(\log L)_{-1}\right\|=\left(\int_{0}^{\infty}\left\{(1+|\log t|)^{-1} f^{* *}(t)\right\}^{q} d t / t\right)^{1 / q} .
$$

Then

$$
L(\infty, q) \cap\left(L_{1}+L_{\infty}\right) \hookrightarrow L^{\infty, q}(\log L)_{-1}, \quad q>1 .
$$

To prove this, we start with (6) for $\gamma=1$ :

$$
f^{* *}(t)=\int_{t}^{1}\left(f^{* *}(s)-f^{*}(s)\right) d s / s+f^{* *}(1) .
$$

Then by the Muckenhoupt inequality [18] for $q>1$,

$$
\left(\int_{0}^{1}\left\{(1+|\log t|)^{-1} f^{* *}(t)\right\}^{q} d t / t\right)^{1 / q} \lesssim\left(\int_{0}^{1}\left(f^{* *}(s)-f^{*}(s)\right)^{q} d s / s\right)^{1 / q}+f^{* *}(1),
$$

and obviously

$$
\left(\int_{1}^{\infty}\left\{(1+|\log t|)^{-1} f^{* *}(t)\right\}^{q} d t / t\right)^{1 / q} \lesssim f^{* *}(1)
$$

Thus (24) is proved.

As a consequence, we obtain the embedding

$$
\tilde{b}_{p, q}^{n / p} \hookrightarrow L^{\infty, q}(\log L)_{-1}, \quad 0<p<\infty, \quad 1<q \leq \infty .
$$

In fact, we have proved a little more, namely

$$
\tilde{b}_{p, q}^{n / p} \hookrightarrow \cap_{w \in W} \Lambda^{q}(w), \quad 0<p<\infty, \quad 1<q \leq \infty,
$$

where the norm in $\Lambda^{q}(w)$ is given by

$$
\left\|f \mid \Lambda^{q}(w)\right\|=\left(\int_{0}^{\infty}\left\{w(t) f^{* *}(t)\right\}^{q} d t / t\right)^{1 / q}
$$

and $W$ is the family of all positive Muckenhoupt weights, that is, weights $w$ satisfying

$$
\left(\int_{0}^{t} w^{q}(s) d s / s\right)^{1 / q} \lesssim(1+|\log t|)^{1 / q-1}, \quad 0<t<1, \quad q>1 .
$$




\section{References}

[1] J. Bastero, M. Milman, and F. J. Ruiz Blasco, A note on $L(\infty, q)$ spaces and Sobolev embeddings, Indiana Univ. Math. J. 52 (2003), no. 5, 1215-1230.

[2] C. Bennett, R. A. De Vore, and R. Sharpley, Weak- $L^{\infty}$ and BMO, Ann. of Math. (2) 113 (1981), no. 3, 601-611.

[3] C. Bennett and R. Sharpley, Interpolation of operators, Pure and Applied Mathematics, vol. 129, Academic Press Inc., Boston, MA, 1988.

[4] J. Bergh and J. Löfström, Interpolation spaces: An introduction, Grundlehren der Mathematischen Wissenschaften, vol. 223, Springer-Verlag, Berlin, 1976.

[5] J. Bourgain, H. Brézis, and P. Mironescu, Limiting embedding theorems for $W^{s, p}$ when $s \uparrow 1$ and applications, J. Anal. Math. 87 (2002), 77-101. Dedicated to the memory of Thomas H. Wolff.

[6] Another look at Sobolev spaces, Optimal Control and Partial Differential Equations (J. L. Menaldi, E. Rofman, and A. Sulem, eds.), IOS Press, 2001. A volume in honour of A. Bensoussan's 60th birthday, pp. 439-455.

[7] M. J. Carro, A. Gogatishvili, J. Martín, and L. Pick, Functional properties of rearrangement invariant spaces defined in terms of oscillations, J. Funct. Anal. 229 (2005), no. 2, 375-404.

[8] D. E. Edmunds, W. D. Evans, and G. E. Karadzhov, Sharp estimates of the embedding constants for Besov spaces, Rev. Mat. Complut. 19 (2006), no. 1, 161-182.

[9] D. E. Edmunds and H. Triebel, Sharp Sobolev embeddings and related Hardy inequalities: The critical case, Math. Nachr. 207 (1999), 79-92.

[10] G. E. Karadzhov, M. Milman, and J. Xiao, Limits of higher-order Besov spaces and sharp reiteration theorems, J. Funct. Anal. 221 (2005), no. 2, 323-339.

[11] V. I. Kolyada and A. K. Lerner, On limiting embeddings of Besov spaces, Studia Math. 171 (2005), no. 1, 1-13.

[12] J. Malý and L. Pick, An elementary proof of sharp Sobolev embeddings, Proc. Amer. Math. Soc. 130 (2002), no. 2, 555-563.

[13] J. Martín and M. Milman, Symmetrization inequalities and Sobolev embeddings, Proc. Amer. Math. Soc. 134 (2006), no. 8, 2335-2347.

[14] _ Higher order symmetrization inequalities and applications, J. Math. Anal. Appl. 330 (2007), 91-113.

[15] V. Maz'ya and T. Shaposhnikova, On the Bourgain, Brezis, and Mironescu theorem concerning limiting embeddings of fractional Sobolev spaces, J. Funct. Anal. 195 (2002), no. 2, 230-238.

[16] _ Erratum to: "On the Bourgain, Brezis and Mironescu theorem concerning limiting embeddings of fractional Sobolev spaces", J. Funct. Anal. 201 (2003), no. 1, 298-300.

[17] M. Milman and E. Pustylnik, On sharp higher order Sobolev embeddings, Commun. Contemp. Math. 6 (2004), no. 3, 495-511.

[18] B. Muckenhoupt, Hardy's inequality with weights, Studia Math. 44 (1972), 31-38.

[19] J. Peetre, Espaces d'interpolation et théorème de Soboleff, Ann. Inst. Fourier (Grenoble) 16 (1966), no. 1, 279-317.

[20] Y. Sagher and P. Shvartsman, Rearrangement-function inequalities and interpolation theory, J. Approx. Theory 119 (2002), no. 2, 214-251.

[21] H. Triebel, Theory of function spaces, Monographs in Mathematics, vol. 78, Birkhäuser Verlag, Basel, 1983.

[22] H. Triebel, Sampling numbers and embedding constants, Tr. Mat. Inst. Steklova 248 (2005), 275-284; English transl., Proc. Steklov Inst. Math. (2005), no. 1 (248), 268-277. 